\title{
Israeli medical students' perceptions of six key medical specialties
}

\author{
Charles Weissman ${ }^{1 *}$, Howard Tandeter ${ }^{5}$, Rachel Yaffa Zisk-Rony ${ }^{4}$, Yoram G Weiss ${ }^{1}$, Uriel Elchalal ${ }^{2}$, Alex Avidan ${ }^{1}$ \\ and Josh E Schroeder ${ }^{3}$
}

\begin{abstract}
Background: Choosing a medical specialty requires medical students to match their interests and social-cultural situations with their perceptions of the various specialties.

Objectives: Examine Israeli 6th-year medical students' perceptions of six key specialties: pediatrics, orthopedic surgery, anesthesiology, obstetrics/gynecology, general surgery and family medicine.

Methods: Questionnaires distributed to 355 6th-year students from three successive classes (2008-2010) of 6th-year students at the Hebrew University - Hadassah School of Medicine, Jerusalem, Israel and the 2010 class of the Ben Gurion University School of Medicine, Be'er Sheva, Israel.

Results: Responses were obtained from 234 students, for a response rate of 66\%. Pediatrics and obstetrics/ gynecology were the specialties most often under positive career consideration by individual students.

Anesthesiology and general surgery were least often under positive career consideration and were viewed as being in a workforce crisis. Pediatrics and family medicine, found to be especially popular among women, were perceived by $58 \%$ and $78 \%$ of respondents, respectively, as providing reasonable ratios of lifestyle to income. None of the students thought the same about general surgery and only $28 \%$ thought so about anesthesiology. Pediatrics and obstetrics/gynecology were reported to afford a controllable lifestyle by $63 \%$ and $8 \%$, respectively, With respect to positive career considerations and lifestyle perceptions, there were no differences between the opinions of men and women students. Differences between genders arose in responses to queries of whether a specialty was interesting and challenging. Women were more likely than men to perceive pediatrics and family medicine as interesting and challenging while men were more likely to think that general and orthopedic surgery are interesting and challenging.

Conclusions: Knowing the medical students' perceptions of the various specialties should help in understanding the maldistribution of physicians among the various specialties. Such data can also be an important input into the efforts of the healthcare leadership to promote a specialty distribution that matches the population's evolving needs.
\end{abstract}

Keywords: Medical education, Residency, Medical specialties, Medical students, Marketing research

\section{Background}

Selecting a career is one of the most important decisions made during a lifetime. The decision requires that individuals match their interests, social situations and cultural backgrounds with a specific vocation. The individual generally gathers information about a number

\footnotetext{
* Correspondence: charles_weissman@yahoo.com

'Department of Anesthesiology and Critical Care Medicine, HadassahHebrew University Medical Center, Hebrew University Hadassah School of Medicine, POB 12000, Jerusalem 91120, Israel

Full list of author information is available at the end of the article
}

of careers from a variety of objective and subjective sources to form an opinion of the occupation [1]. Medical students must make a two or three-step career decision. The initial decision is the educational choice to become a physician and attend medical school [1]. The second decision is the occupational choice of one of the more than 20 medical specialties for residency training. A further step is deciding whether to pursue further training and, if yes, in which subspecialty.

The medical specialties differ greatly in work settings, skill sets, duties, responsibilities and professional interests,

\section{Biomed Central}


so that they essentially constitute a group of distinct occupations [2,3]. Therefore, choosing a specialty is complicated and made even more problematic by modern medical care becoming increasingly complex, resulting in increasing specialization and sub-specialization. Simultaneously, as populations continue to grow, so does the demand for more primary care physicians. The distribution of physicians among the primary, secondary and tertiary specialties is a function of the number of medical school graduates, their choice of specialties, and the number of available residency and fellowship training positions.

Israel is beset by a looming shortage of physicians, an inadequate number of medical school graduates and a maldistribution of physicians among the various specialties, with shortages in areas such as general surgery and anesthesiology. This problematic situation requires healthcare system leadership to gain additional insights into issues concerning the physician workforce, such as medical students' perceptions of the various specialties. Knowing these perceptions is especially important for discerning why some specialties are more popular than others among the medical students.

This study is a component of a larger investigation of the processes Israeli medical students utilize when selecting medical specialties $[4,5]$. It specifically explores a key component in the second decision step enumerated above (i.e. decisions about which specialty to pursue), namely final (6th) year medical students' perceptions of six key specialties: (pediatrics, orthopedic surgery, anesthesiology, obstetrics/gynecology, general surgery, and family medicine). The study examines student perceptions of both the professional and non-professional aspects of these specialties. The interest in the latter is due to the many studies showing the importance that the current generation of medical students attaches to balancing professional obligations with free-time (child-rearing, family time) and a controllable lifestyle with financial compensation [6-8]. The objective of the study was to better understand how and why medical students, both male and female, would choose or reject one of these specialties for residency training and career.

\section{Methods}

The methods used in this study were adapted from marketing research methods and have been described elsewhere [5]. Briefly, this methodology proposes that when a consumer's (i.e. student's) criteria match his/her perceptions of a product's (i.e. specialty) features, the likelihood of a purchase (selecting the specialty) increases. Therefore, to provide insights into the selection process, this study examined the two sides of the marketing equation: both the students' selection criteria and their perceptions of the various specialties. This was accomplished using a questionnaire designed to elicit (1) the importance of each of the 25 criteria with respect to the students' choice of a medical specialty; (2) their perceptions (16 items) of how six key specialties (pediatrics, orthopedic surgery, anesthesiology, obstetrics/gynecology, general surgery and family medicine) rate on each of the 25 criteria; (3) their level of consideration in pursuing a career in each of these specialties; and (4) demographic data. A 5-point Likert scale was used for answering the questions related to the first three items in this list.

The study focused on four specialties mirroring those practiced by the investigators (family medicine, obstetrics/ gynecology, orthopedic surgery and anesthesiology) plus pediatrics and general surgery. This provided information on two primary care specialties (pediatrics and family practice) and two surgical specialties (general and orthopedic surgery) plus obstetrics/gynecology and anesthesiology. General Internal Medicine was not chosen because Internal Medicine is usually a "pass through" residency i.e. a way-station on the way to sub-specialization. Only a minority of Israeli internal medicine residents do not sub-specialize ${ }^{\mathrm{a}}$.

The questionnaires underwent two pilot studies to examine ease-of-use and determine whether modifications were needed. Each of these initial studies included fifteen 6th-year medical students. During the pilot studies, information was elicited on 17 items for each of 10 specialties. However, many of the respondents failed to answer all the questions because of the excessive time it was taking to complete the forms. Therefore, the number of specialties was reduced to six to provide information on two primary care specialties (pediatrics and family practice) and two surgical specialties (general and orthopedic surgery) plus obstetrics/gynecology and anesthesiology [5].

The questionnaire's final version was distributed to three successive classes (2008-2010) of 6th-year students at the Hebrew University - Hadassah School of Medicine, Jerusalem, Israel and the 2010 class of the Ben Gurion University School of Medicine, Be'er Sheva, Israel. These two schools were chosen because they are the investigators' home institutions. This allowed them to insure a high response rate.

Portions of these data have been reported in a methodological validation (5). This study was approved by the Institutional Review Board of the Hadassah Medical Organization.

For statistical analysis, all the points on the Likert Scale were treated as continuous variables. The Likert Scale data were subjected to both hierarchal cluster analysis and factor analysis. In the latter, varimax rotation was used with set eigenvalues of $\geq 1.0$. When reported as categorical data, the 5-points of the Likert Scale were reduced to three categories (the percentages of the responses from the two points representing negative responses were added together as were the percentages of two points representing positive 
responses plus the middle point). The percentage of responses for each of the three categories was calculated.

Analysis focused on two issues. (1) The students' perceptions of each of the six specialties and how the perception patterns differed between the various specialties. (2) The differences between the selection criteria and perceptions of students considering and not considering pursuing a career in each of the specialties. For this analysis the replies of the students who chose one of the two negative points on the 5-point Likert Scale to the query "are you considering a career in this specialty" were compared to those of the students who chose one of the two positive points; i.e. the neutral choice was not included in the analysis.

These data were managed with Excel $2003^{\circ}$ (Microsoft Inc., Redmont, WA) and analyzed using Systat Version $12^{\circ}$ (Systat Inc. San Jose, CA). Categorical data are presented as frequency distributions. Chi-squared analysis was performed for binomial responses. Two-tailed Student's t-tests compared continuous variables and Bonferroni corrections were employed for multiple comparisons. Based on prior investigations demonstrating significant differences in interests and selection criteria between the genders $[9,10]$, it was decided during the study's design phase to separately evaluate data from male and female students.

\section{Results}

A response rate of $66 \%$ (234 of 355 questionnaires) was obtained. Of the respondents, $52 \%$ were women and $53 \%$ were single. The male students were older $(\mathrm{p}<0.001)$ than the female ones.

As indicated in Figures 1 and 2, the selection criteria rated most highly were "interesting and challenging specialty", "family time", and "controllable lifestyle". The criterion rated lowest was "work limited hours", while "high salary" and "private practice" received intermediate ratings.

The medical students' perceptions of all six specialties are found in Table 1, which displays the percentage of agree/highly agree replies on the 5-point Likert Scale. Each specialty had a distinct profile. Pediatrics and obstetrics/gynecology were the specialties most often reported as being under positive consideration for a career both by the students themselves and by their peers. Anesthesiology and general surgery were least often being positively considered as careers by the students and both were thought to be in workforce crises. There were no differences in the findings between the two schools.

Hierarchal cluster analysis revealed the following cluster of criteria: controllable lifestyle, family time, and working limited hours. Factor analysis included the following factors: (1) working limited hours and family time with long working hours as a reciprocal or inverse member ${ }^{\text {b }}$ (2) high salary and specialty being highly considered by colleagues; and (3) long working hours with reciprocal member, the ratio of lifestyle to income.

Only a few differences were found between the perceptions of male and female students of each of the specialties (Table 2). The women students had a more positive view of pediatrics and family medicine than the men. Conversely, the men had a more positive perception of orthopedic surgery. Perceiving a specialty as interesting and challenging was a major determinant of positively considering a specialty.

The differences between the perceptions and selection criteria of students considering and not considering pursuing a career in each of the six specialties are found on

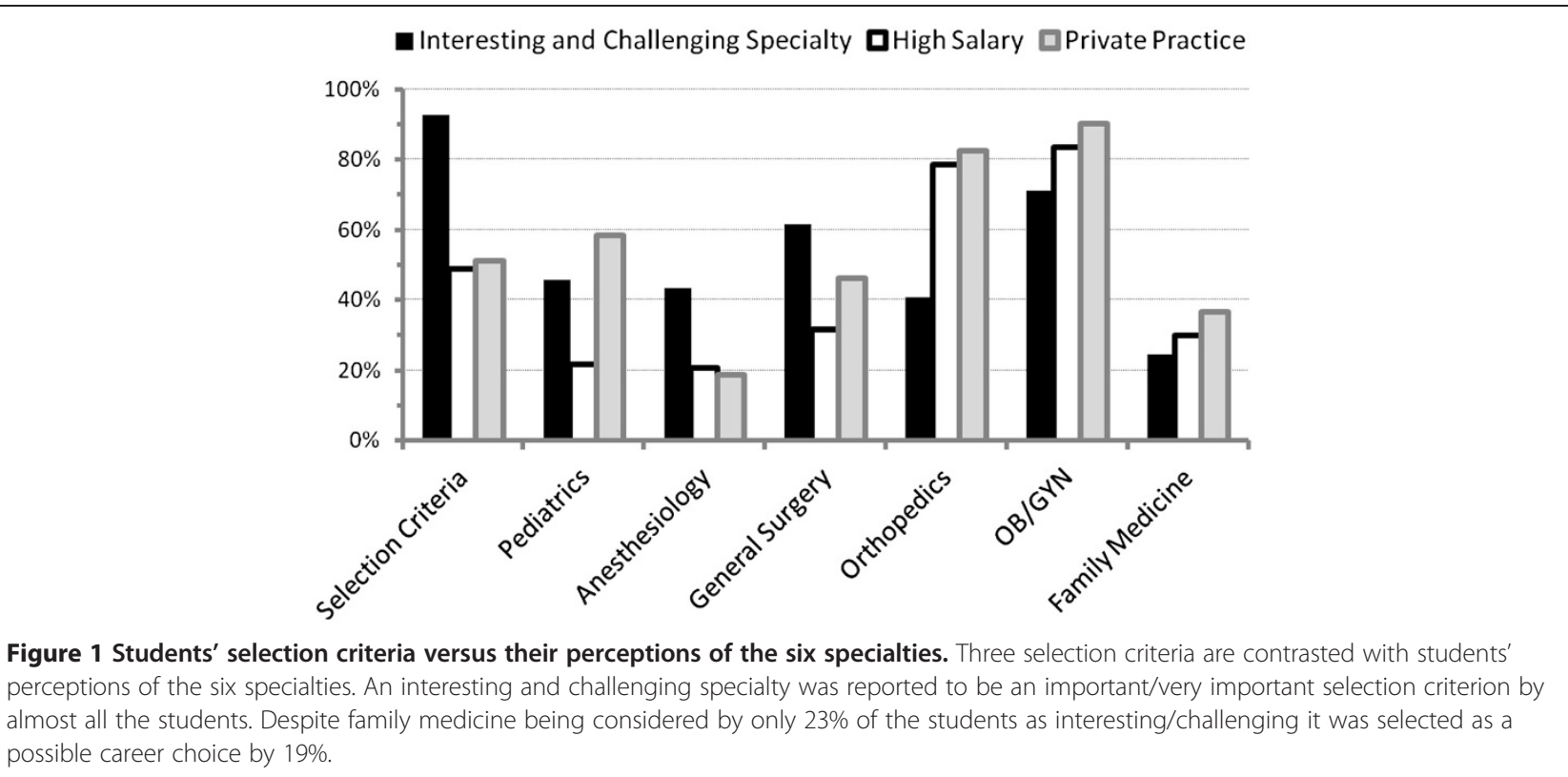




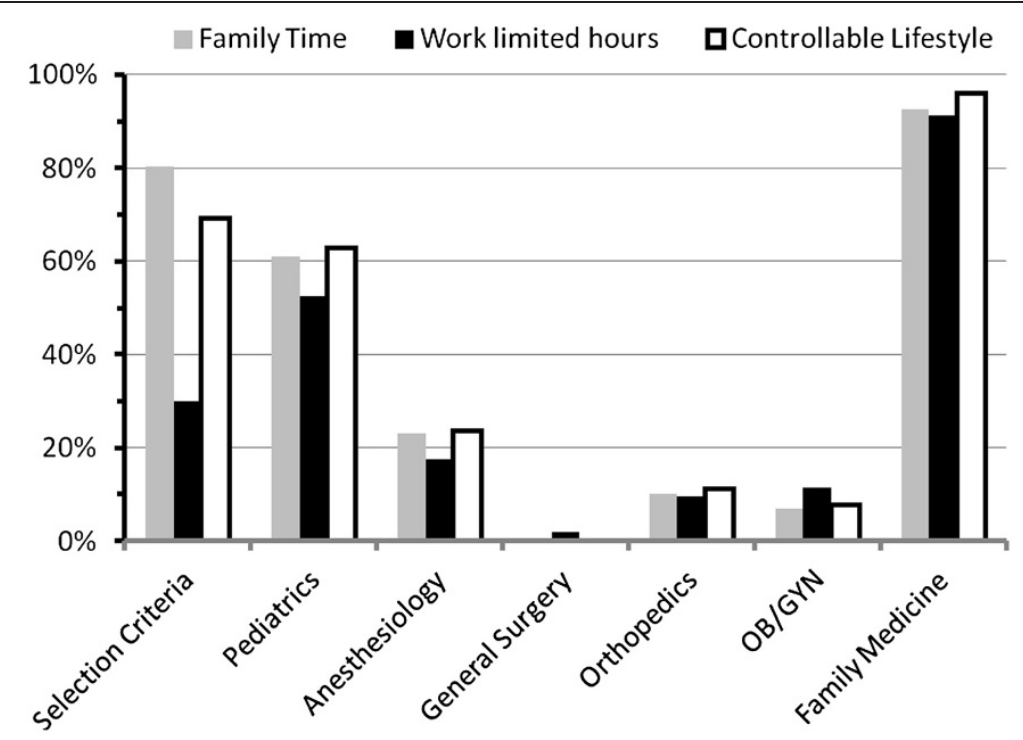

Figure 2 Students' selection criteria versus their perceptions of the six specialties. Three of the selection criteria are contrasted with the students' perceptions of corresponding factors. Both a controllable lifestyle and family time were considered important/very important selection criteria by many students. Pediatrics and family medicine were considered to afford both controllable lifestyles and family time.

Table 1 Medical students' perceptions of the six specialties $(n=234)$

\begin{tabular}{|c|c|c|c|c|c|c|}
\hline & Pediatrics & Anesthesiology & $\begin{array}{l}\text { General } \\
\text { Surgery }\end{array}$ & $\begin{array}{l}\text { Orthopedic } \\
\text { Surgery }\end{array}$ & OB/GYN & $\begin{array}{l}\text { Family } \\
\text { Medicine }\end{array}$ \\
\hline Interesting and challenging specialty & $46 \%$ & $43 \%$ & $62 \% \neq$ & $41 \%+$ & $71 \% \S^{*} \neq \dagger$ & $25 \%{ }^{*} \neq \dagger a$ \\
\hline Advanced specialty & $36 \%$ & $44 \% *$ & $35 \% \%^{*} \neq$ & $52 \% *+$ & $69 \% *+$ & $23 \%^{*} \neq \dagger \S^{a}$ \\
\hline Boring specialty & $21 \%$ & $35 \% *$ & $14 \% \neq$ & $30 \% *+$ & $7 \% * \neq+\S$ & $48 \% \%^{*}+a$ \\
\hline Stressful specialty & $27 \%$ & $74 \% *$ & $94 \% * \neq$ & $48 \%{ }^{*} \neq \dagger$ & $81 \% *+\S$ & $13 \%{ }^{*} \neq \dagger \S^{a}$ \\
\hline Affords controllable lifestyle & $63 \%$ & $24 \% *$ & $0 \% * \neq$ & $11 \% *+$ & $8 \% *+$ & $96 \%{ }^{*} \neq+\S^{a}$ \\
\hline Possible to work limited hours & $53 \%$ & $17 \% *$ & $2 \% * \neq$ & $10 \% *+$ & $12 \% *+$ & $91 \%{ }^{*} \neq+\S^{a}$ \\
\hline Allows for family time & $61 \%$ & $23 \% *$ & $0 \%{ }^{*} \neq$ & $10 \%{ }^{*} \dagger$ & $7 \% *+$ & $93 \%^{*} \neq+\S^{a}$ \\
\hline Long working hours & $25 \%$ & $62 \% *$ & $97 \% * \neq$ & $75 \% *+$ & $86 \% \%^{*}+\dagger$ & $6 \%{ }^{*} \neq+\S^{a}$ \\
\hline Prestigious specialty (population) & $33 \%$ & $5 \% *$ & $78 \%{ }^{*} \neq$ & $49 \% * \neq 十$ & $71 \% * \ddagger \S$ & $7 \% * \S^{a}$ \\
\hline Prestigious specialty (colleagues) & $15 \%$ & $10 \% *$ & $46 \% * \neq$ & $34 \% *+$ & $61 \% * \neq \dagger$ & $2 \%^{*}+\dagger \S^{a}$ \\
\hline Opportunity for private practice & $58 \%$ & $19 \% *$ & $46 \% * \neq$ & $83 \% \%^{*} \neq \dagger$ & $90 \% *+十$ & $37 \%^{*} \neq+\S^{a}$ \\
\hline High salary & $22 \%$ & $21 \% *$ & $32 \% * \neq$ & $79 \%{ }^{*} \neq \dagger$ & $84 \% *+十$ & $30 \% \neq \S^{a}$ \\
\hline Reasonable ratio of lifestyle vs income & $58 \%$ & $20 \% *$ & $6 \% * \neq$ & $53 \% \neq \dagger$ & $45 \% \neq \dagger$ & $78 \%{ }^{*} \neq+\S^{a}$ \\
\hline Academic opportunities & $45 \%$ & $46 \%$ & $58 \%$ & $46 \%$ & $78 \% * \neq \dagger$ & $8 \%{ }^{*} \neq \dagger \S^{a}$ \\
\hline Specialty other students positively consider & $67 \%$ & $1 \% *$ & $6 \% * \neq$ & $50 \% * \neq \dagger$ & $76 \% \neq+\S$ & $32 \% * \neq+a$ \\
\hline Specialty in crisis & $7 \%$ & $97 \% *$ & $74 \% * \neq$ & $3 \% \neq \dagger$ & $3 \% \neq \dagger$ & $16 \% \neq † \S а$ \\
\hline Specialty I am positively considering & $35 \%$ & $9 \%^{*}$ & $20 \% *$ & $16 \% *$ & $34 \% \neq † \S$ & $19 \%{ }^{*} \neq a$ \\
\hline * $p<0.001$ vs pediatrics & & $\begin{array}{l}\text { Values are the percentage } \\
\text { of agree/highly agree answers } \\
\text { on a } 5 \text { point-Likert Scale }\end{array}$ & & & & \\
\hline
\end{tabular}

$\neq \mathrm{p}<0.001$ vs anesthesiology.

$\dagger \mathrm{p}<0.001$ vs surgery.

$\S p<0.001$ vs orthopedics.

a $p<0.001$ vs OB/GYN. 
Table 2 Gender differences in the perceptions of the six specialties (Women: $n=122 ;$ Men: $n=112$ )

\begin{tabular}{|c|c|c|c|c|c|c|}
\hline & $\begin{array}{l}\text { Males } \\
\text { Pediatrics }\end{array}$ & $\begin{array}{l}\text { Females } \\
\text { Pediatrics }\end{array}$ & $\begin{array}{l}\text { Males } \\
\text { Anesthesiology }\end{array}$ & $\begin{array}{l}\text { Females } \\
\text { Anesthesiology }\end{array}$ & $\begin{array}{l}\text { Males } \\
\text { General }\end{array}$ & $\begin{array}{l}\text { Females } \\
\text { General }\end{array}$ \\
\hline & & & & & Surgery & Surgery \\
\hline Interesting and challenging specialty & $38 \%$ & $53 \% *$ & $45 \%$ & $42 \%$ & $73 \%$ & $51 \% *$ \\
\hline Advanced specialty & $34 \%$ & $37 \%$ & $39 \%$ & $48 \%$ & $37 \%$ & $33 \%$ \\
\hline Boring specialty & $31 \%$ & $12 \%^{*}$ & $37 \%$ & $34 \%$ & $11 \%$ & $16 \%$ \\
\hline Stressful specialty & $27 \%$ & $26 \%$ & $70 \%$ & $78 \%$ & $95 \%$ & $94 \%$ \\
\hline Affords a controllable lifestyle & $62 \%$ & $64 \%$ & $28 \%$ & $20 \%$ & $0 \%$ & $0 \%$ \\
\hline Possibility to work limited hours & $56 \%$ & $50 \%$ & $21 \%$ & $14 \%$ & $1 \%$ & $3 \%$ \\
\hline Allows for family time & $60 \%$ & $62 \%$ & $29 \%$ & $18 \%$ & $0 \%$ & $0 \%$ \\
\hline Long working hours & $30 \%$ & $21 \%$ & $57 \%$ & $67 \% *$ & $99 \%$ & $96 \%$ \\
\hline Prestigious specialty (population) & $30 \%$ & $36 \%$ & $5 \%$ & $4 \%$ & $75 \%$ & $80 \%$ \\
\hline Prestigious specialty (colleagues) & $17 \%$ & $13 \%$ & $10 \%$ & $11 \%$ & $50 \%$ & $43 \%$ \\
\hline Opportunity for private practice & $59 \%$ & $58 \%$ & $18 \%$ & $22 \%$ & $51 \%$ & $42 \%$ \\
\hline High salary & $23 \%$ & $21 \%$ & $19 \%$ & $22 \%$ & $30 \%$ & $33 \%$ \\
\hline Reasonable ratio of lifestyle to income & $57 \%$ & $58 \%$ & $22 \%$ & $19 \%$ & $4 \%$ & $8 \%$ \\
\hline Academic opportunities & $50 \%$ & $41 \%$ & $45 \%$ & $47 \%$ & $63 \%$ & $55 \%$ \\
\hline Specialty in crisis & $8 \%$ & $6 \%$ & $97 \%$ & $96 \%$ & $81 \%$ & $68 \%$ \\
\hline Specialty other students positively consider & $69 \%$ & $66 \%$ & $0 \%$ & $1 \% *$ & $7 \%$ & $5 \%$ \\
\hline \multirow[t]{4}{*}{ Specialty I am positively considering } & $24 \%$ & $45 \% *$ & $14 \%$ & $5 \% *$ & $32 \%$ & $9 \% *$ \\
\hline & Males & Females & Males & Females & Males & Females \\
\hline & Orthopedic & Orthopedic & Obstetrics/ & Obstetrics/ & Family & Family \\
\hline & Surgery & Surgery & Gynecology & Gynecology & Medicine & Medicine \\
\hline Interesting and challenging specialty & $53 \%$ & $30 \% *$ & $64 \%$ & $78 \% *$ & $22 \%$ & $27 \% *$ \\
\hline Advanced specialty & $58 \%$ & $46 \% *$ & $63 \%$ & $76 \% *$ & $22 \%$ & $23 \%$ \\
\hline Boring specialty & $23 \%$ & $36 \%$ & $7 \%$ & $8 \%$ & $52 \%$ & $44 \% *$ \\
\hline Stressful specialty & $51 \%$ & $45 \%$ & $78 \%$ & $83 \%$ & $13 \%$ & $14 \% *$ \\
\hline Affords a controllable lifestyle & $14 \%$ & $9 \%$ & $8 \%$ & $8 \%$ & $96 \%$ & $95 \%$ \\
\hline Possibility to work limited hours & $9 \%$ & $10 \%$ & $10 \%$ & $13 \%$ & $90 \%$ & $93 \%$ \\
\hline Allows for family time & $11 \%$ & $9 \%$ & $6 \%$ & $8 \%$ & $91 \%$ & $94 \%$ \\
\hline Long working hours & $79 \%$ & $72 \%$ & $83 \%$ & $88 \%$ & $6 \%$ & $5 \%$ \\
\hline Prestigious specialty (population) & $48 \%$ & $50 \%$ & $64 \%$ & $78 \% *$ & $6 \%$ & $9 \%$ \\
\hline Prestigious specialty (colleagues) & $34 \%$ & $35 \%$ & $53 \%$ & $69 \% *$ & $3 \%$ & $1 \%$ \\
\hline Opportunities for private practice & $87 \%$ & $78 \%$ & $92 \%$ & $87 \%$ & $36 \%$ & $37 \%$ \\
\hline High salary & $80 \%$ & $77 \%$ & $87 \%$ & $69 \%$ & $30 \%$ & $31 \%$ \\
\hline Reasonable ratio of lifestyle to income & $55 \%$ & $51 \%$ & $48 \%$ & $42 \%$ & $78 \%$ & $77 \%$ \\
\hline Academic opportunities & $48 \%$ & $45 \%$ & $79 \%$ & $76 \%$ & $9 \%$ & $10 \%$ \\
\hline Specialty in crisis & $3 \%$ & $4 \%$ & $3 \%$ & $3 \%$ & $17 \%$ & $15 \%$ \\
\hline Specialty other students positively consider & $58 \%$ & $44 \% *$ & $75 \%$ & $78 \%$ & $27 \%$ & $36 \% *$ \\
\hline Specialty I am positively considering & $28 \%$ & $6 \% *$ & $32 \%$ & $36 \%$ & $13 \%$ & $25 \% *$ \\
\hline
\end{tabular}

* vs males $p<0.05$.

Values are the percentage of agree/highly agree answers on a 5 point-Likert Scale.

Table 3. The research not only provides insight into the students' views of each of the specialties, but also the interactions between their selection criteria, perceptions, and interest in pursuing a specific specialty as a career.
For example, a specialty providing an opportunity for private practice was deemed an important/very important selection criterion by $51 \%$ of respondents (Figure 1), while orthopedic surgery and obstetrics/gynecology were 
Table 3 Perceptions and selection criteria of students considering and not considering pursuing a career in each of the specialties

\begin{tabular}{|c|c|c|c|c|c|c|c|c|c|}
\hline & \multicolumn{2}{|c|}{ Pediatrics } & & \multicolumn{3}{|c|}{ Anesthesiology } & \multicolumn{3}{|c|}{ General surgery } \\
\hline & Yes & No & & Yes & No & & Yes & No & \\
\hline \multirow[t]{2}{*}{ Specialty I am positively considering } & $(n=75)$ & $(n=99)$ & & $(n=21)$ & $(n=164)$ & & $(n=41)$ & $(n=142)$ & \\
\hline & $32 \%$ & $42 \%$ & & $9 \%$ & $70 \%$ & & $18 \%$ & $61 \%$ & \\
\hline Interesting and challenging specialty & $76 \%$ & $22 \%$ & $p<0.001$ & $90 \%$ & $34 \%$ & $p<0.001$ & $90 \%$ & $51 \%$ & $p<0.001$ \\
\hline Advanced specialty & $57 \%$ & $23 \%$ & $p<0.001$ & $67 \%$ & $40 \%$ & $p<0.003$ & $38 \%$ & $33 \%$ & NS \\
\hline Boring specialty & $7 \%$ & $36 \%$ & $p<0.001$ & $21 \%$ & $42 \%$ & $p=0.003$ & $5 \%$ & $19 \%$ & $p<0.001$ \\
\hline Stressful specialty & $36 \%$ & $18 \%$ & $p<0.023$ & $84 \%$ & $72 \%$ & NS & $95 \%$ & $94 \%$ & NS \\
\hline Controllable lifestyle & $68 \%$ & $66 \%$ & NS & $35 \%$ & $26 \%$ & NS & $0 \%$ & $0 \%$ & NS \\
\hline Work limited hours & $51 \%$ & $58 \%$ & NS & $26 \%$ & $19 \%$ & NS & $2 \%$ & $2 \%$ & NS \\
\hline Allows family time & $64 \%$ & $63 \%$ & NS & $37 \%$ & $24 \%$ & NS & $0 \%$ & $0 \%$ & NS \\
\hline Long working hours & $57 \%$ & $23 \%$ & $p<0.001$ & $67 \%$ & $40 \%$ & $p<0.003$ & $38 \%$ & $33 \%$ & NS \\
\hline Prestigious specialty (population) & $36 \%$ & $27 \%$ & $p<0.05$ & $11 \%$ & $4 \%$ & NS & $76 \%$ & $79 \%$ & NS \\
\hline Prestigious specialty (colleagues) & $22 \%$ & $10 \%$ & $p=0.03$ & $26 \%$ & $8 \%$ & NS & $63 \%$ & $42 \%$ & $p=0.048$ \\
\hline Opportunities for private practice & $59 \%$ & $60 \%$ & NS & $32 \%$ & $16 \%$ & NS & $54 \%$ & $42 \%$ & NS \\
\hline High salary & $20 \%$ & $21 \%$ & NS & $21 \%$ & $21 \%$ & NS & $34 \%$ & $31 \%$ & NS \\
\hline Ratio of lifestyle vs income & $65 \%$ & $54 \%$ & NS & $28 \%$ & $20 \%$ & NS & $5 \%$ & $6 \%$ & NS \\
\hline Academic opportunities & $45 \%$ & $45 \%$ & NS & $42 \%$ & $43 \%$ & NS & $68 \%$ & $55 \%$ & NS \\
\hline Specialty in crisis & $8 \%$ & $9 \%$ & NS & $95 \%$ & $97 \%$ & NS & $73 \%$ & $74 \%$ & NS \\
\hline Requested specialty & $77 \%$ & $56 \%$ & $p=0.007$ & $0 \%$ & $0 \%$ & NS & $17 \%$ & $4 \%$ & NS \\
\hline \multicolumn{10}{|l|}{ Selection criteria } \\
\hline Bedside specialty & $80 \%$ & $66 \%$ & $p=0.004$ & $63 \%$ & $71 \%$ & NS & $63 \%$ & $76 \%$ & NS \\
\hline Direct aid to patients & $77 \%$ & $77 \%$ & NS & $76 \%$ & $78 \%$ & NS & $85 \%$ & $74 \%$ & NS \\
\hline Direct patient care & $69 \%$ & $55 \%$ & $p=0.02$ & $43 \%$ & $65 \%$ & NS & $44 \%$ & $66 \%$ & $p=0.009$ \\
\hline Time for childrearing & $87 \%$ & $58 \%$ & $p<0.001$ & $71 \%$ & $72 \%$ & NS & $49 \%$ & $81 \%$ & $p<0.001$ \\
\hline Family time & $92 \%$ & $69 \%$ & $p<0.001$ & $80 \%$ & $80 \%$ & NS & $56 \%$ & $88 \%$ & $p<0.001$ \\
\hline Only daytime work & $44 \%$ & $18 \%$ & $p<0.001$ & $19 \%$ & $29 \%$ & NS & $7 \%$ & $38 \%$ & $p<0.001$ \\
\hline Work outside the hospital & $11 \%$ & $8 \%$ & NS & $14 \%$ & $11 \%$ & NS & $2 \%$ & $12 \%$ & $p<0.001$ \\
\hline Long-term care & $44 \%$ & $32 \%$ & NS & $24 \%$ & $46 \%$ & NS & $15 \%$ & $49 \%$ & $p=0.002$ \\
\hline Family orientation & $45 \%$ & $28 \%$ & $p=0.002$ & $26 \%$ & $39 \%$ & NS & $15 \%$ & $42 \%$ & $p=0.002$ \\
\hline Immediate satisfaction & $49 \%$ & $61 \%$ & NS & $79 \%$ & $50 \%$ & NS & $90 \%$ & $42 \%$ & $p<0.001$ \\
\hline Operating room time & $23 \%$ & $53 \%$ & $p<0.001$ & $29 \%$ & $38 \%$ & NS & $71 \%$ & $24 \%$ & $p<0.001$ \\
\hline Performing procedures & $35 \%$ & $61 \%$ & $p<0.001$ & $57 \%$ & $46 \%$ & NS & $90 \%$ & $33 \%$ & $p<0.001$ \\
\hline Controllable lifestyle & $68 \%$ & $65 \%$ & NS & $67 \%$ & $69 \%$ & NS & $46 \%$ & $79 \%$ & $p=0.004$ \\
\hline "Action" specialty & $25 \%$ & $48 \%$ & $p<0.002$ & $43 \%$ & $36 \%$ & NS & $73 \%$ & $25 \%$ & $p<0.001$ \\
\hline Without long-term care & $11 \%$ & $13 \%$ & NS & $24 \%$ & $10 \%$ & NS & $12 \%$ & $11 \%$ & NS \\
\hline On-cal as an attending & $41 \%$ & $33 \%$ & NS & $33 \%$ & $35 \%$ & NS & $44 \%$ & $34 \%$ & $p=0.008$ \\
\hline High salary & $47 \%$ & $48 \%$ & NS & $62 \%$ & $45 \%$ & NS & $49 \%$ & $46 \%$ & NS \\
\hline Private practice & $47 \%$ & $56 \%$ & NS & $38 \%$ & $50 \%$ & NS & $51 \%$ & $49 \%$ & NS \\
\hline Prestigious specialty & $8 \%$ & $15 \%$ & $p<0.003$ & $5 \%$ & $12 \%$ & $p<0.037$ & $27 \%$ & $8 \%$ & $p=0.002$ \\
\hline Prestigious specialty & $8 \%$ & $14 \%$ & NS & $5 \%$ & $10 \%$ & NS & $24 \%$ & $7 \%$ & $p=0.007$ \\
\hline Interesting specialty & $91 \%$ & $93 \%$ & NS & $81 \%$ & $93 \%$ & NS & $93 \%$ & $91 \%$ & NS \\
\hline Gender (Female) & $68 \%$ & $42 \%$ & $p<0.001$ & $29 \%$ & $52 \%$ & $p<0.043$ & $24 \%$ & $62 \%$ & \\
\hline Marital status (Single) & $47 \%$ & $55 \%$ & NS & & & NS & $52 \%$ & $59 \%$ & NS \\
\hline
\end{tabular}


Table 3 Perceptions and selection criteria of students considering and not considering pursuing a career in each of the specialties (Continued)

\begin{tabular}{|c|c|c|c|c|c|c|c|c|c|}
\hline & \multicolumn{3}{|c|}{ Orthopedic surgery } & \multicolumn{3}{|c|}{ Obstetrics/gynecology } & \multicolumn{3}{|c|}{ Family medicine } \\
\hline & Yes & No & & Yes & No & & Yes & No & \\
\hline \multirow[t]{2}{*}{ Specialty I am positively considering } & $(n=34)$ & $(n=155))$ & & $(n=74)$ & $(n=102)$ & & $(n=41)$ & $(n=137)$ & \\
\hline & $15 \%$ & $66 \%$ & & $32 \%$ & $44 \%$ & & $18 \%$ & $59 \%$ & \\
\hline Interesting and challenging specialty & $88 \%$ & $27 \%$ & $p<0.001$ & $93 \%$ & $55 \%$ & $p<0.001$ & $68 \%$ & $9 \%$ & $p<0.001$ \\
\hline Advanced specialty & $68 \%$ & $47 \%$ & $p=0.036$ & $84 \%$ & $60 \%$ & $p<0.001$ & $29 \%$ & $23 \%$ & NS \\
\hline Boring specialty & $6 \%$ & $39 \%$ & $p<0.001$ & $3 \%$ & $12 \%$ & $p<0.001$ & $18 \%$ & $65 \%$ & $P<0.001$ \\
\hline Stressful specialty & $44 \%$ & $50 \%$ & NS & $88 \%$ & $80 \%$ & NS & $12 \%$ & $15 \%$ & NS \\
\hline Controllable lifestyle & $26 \%$ & $7 \%$ & $p=0.011$ & $9 \%$ & $7 \%$ & NS & $100 \%$ & $94 \%$ & NS \\
\hline Work limited hours & $12 \%$ & $7 \%$ & NS & $11 \%$ & $9 \%$ & NS & $93 \%$ & $90 \%$ & NS \\
\hline Allows family time & $18 \%$ & $8 \%$ & $p=0.043$ & $7 \%$ & $6 \%$ & NS & $93 \%$ & $92 \%$ & NS \\
\hline Long working hours & $85 \%$ & $74 \%$ & NS & $89 \%$ & $84 \%$ & NS & $15 \%$ & $4 \%$ & $P=0.028$ \\
\hline Prestigious specialty (population) & $59 \%$ & $47 \%$ & NS & $71 \%$ & $67 \%$ & NS & $8 \%$ & $7 \%$ & NS \\
\hline Prestigious specialty (colleagues) & $26 \%$ & $38 \%$ & NS & $64 \%$ & $57 \%$ & NS & $0 \%$ & $3 \%$ & NS \\
\hline Opportunities for private practice & $97 \%$ & $79 \%$ & $p<0.001$ & $88 \%$ & $88 \%$ & NS & $30 \%$ & $41 \%$ & NS \\
\hline -High salary & $82 \%$ & $77 \%$ & NS & $84 \%$ & $81 \%$ & NS & $33 \%$ & $31 \%$ & NS \\
\hline Reasnable ratio of lifestyle vs. income & $62 \%$ & $50 \%$ & $p=0.042$ & $51 \%$ & $39 \%$ & NS & $93 \%$ & $73 \%$ & $p=0.004$ \\
\hline Academic opportunities & $62 \%$ & $42 \%$ & NS & $81 \%$ & $74 \%$ & NS & $15 \%$ & $6 \%$ & $P=0.012$ \\
\hline Specialty in crisis & $6 \%$ & $3 \%$ & NS & $1 \%$ & $4 \%$ & NS & $26 \%$ & $15 \%$ & NS \\
\hline Requested specialty & $62 \%$ & $45 \%$ & $p=0.018$ & $73 \%$ & $73 \%$ & NS & $40 \%$ & $28 \%$ & NS \\
\hline \multicolumn{10}{|l|}{ Selecion criteria } \\
\hline Bedside specialty & $53 \%$ & $77 \%$ & NS & $74 \%$ & $74 \%$ & NS & $85 \%$ & $72 \%$ & $p=0.036$ \\
\hline Direct aid to patients & $76 \%$ & $77 \%$ & NS & $76 \%$ & $75 \%$ & NS & $80 \%$ & $79 \%$ & NS \\
\hline Direct patient care & $35 \%$ & $68 \%$ & $p=0.004$ & $57 \%$ & $65 \%$ & NS & $78 \%$ & $57 \%$ & $p=0.042$ \\
\hline Time for childrearing & $50 \%$ & $77 \%$ & $p=0.023$ & $66 \%$ & $73 \%$ & NS & $95 \%$ & $63 \%$ & $p<0.001$ \\
\hline Family time & $62 \%$ & $84 \%$ & $p=0.016$ & $76 \%$ & $79 \%$ & NS & $95 \%$ & $74 \%$ & $p<0.001$ \\
\hline Only daytime work & $15 \%$ & $34 \%$ & $p=0.001$ & $26 \%$ & $29 \%$ & NS & $51 \%$ & $20 \%$ & $p<0.001$ \\
\hline Work outside the hospital & $6 \%$ & $12 \%$ & $p=0.046$ & $7 \%$ & $12 \%$ & NS & $24 \%$ & $5 \%$ & $p<0.001$ \\
\hline Long-term care & $15 \%$ & $45 \%$ & $p<0.001$ & $41 \%$ & $35 \%$ & NS & $68 \%$ & $28 \%$ & $p<0.001$ \\
\hline Family orientation & $15 \%$ & $39 \%$ & $p=0.003$ & $36 \%$ & $31 \%$ & NS & $70 \%$ & $24 \%$ & $p<0.001$ \\
\hline Immediate satisfaction & $79 \%$ & $46 \%$ & $p<0.001$ & $70 \%$ & $48 \%$ & $p<0.001$ & $34 \%$ & $63 \%$ & $p=0.003$ \\
\hline Operating room time & $74 \%$ & $28 \%$ & $p<0.001$ & $53 \%$ & $33 \%$ & $p<0.001$ & $17 \%$ & $46 \%$ & $p<0.001$ \\
\hline Performing procedures & $85 \%$ & $39 \%$ & $p<0.001$ & $58 \%$ & $41 \%$ & NS & $24 \%$ & $55 \%$ & $p<0.001$ \\
\hline Controllable lifestyle & $68 \%$ & $70 \%$ & NS & $59 \%$ & $74 \%$ & NS & $83 \%$ & $62 \%$ & NS \\
\hline "Action" specialty & $59 \%$ & $31 \%$ & $p<0.001$ & $45 \%$ & $34 \%$ & $p<0.008$ & $15 \%$ & $48 \%$ & $p<0.001$ \\
\hline Without long-term care & $9 \%$ & $12 \%$ & NS & $11 \%$ & $11 \%$ & NS & $10 \%$ & $12 \%$ & NS \\
\hline On-cal as an attending & $38 \%$ & $37 \%$ & $p=0.029$ & $37 \%$ & $38 \%$ & NS & $27 \%$ & $44 \%$ & $p=0.006$ \\
\hline High salary & $62 \%$ & $43 \%$ & $p=0.024$ & $53 \%$ & $42 \%$ & NS & $46 \%$ & $47 \%$ & NS \\
\hline Private practice & $62 \%$ & $45 \%$ & $\mathrm{p}<0.001$ & $58 \%$ & $43 \%$ & $p<0.004$ & $34 \%$ & $56 \%$ & $p=0.022$ \\
\hline Prestigious specialty (colleagues) & $18 \%$ & $9 \%$ & NS & $13 \%$ & $12 \%$ & NS & $3 \%$ & $18 \%$ & $p=0.002$ \\
\hline Prestigious specialty (population) & $53 \%$ & $48 \%$ & NS & $11 \%$ & $11 \%$ & NS & $2 \%$ & $16 \%$ & $p=0.039$ \\
\hline Interesting specialty & $91 \%$ & $92 \%$ & NS & $92 \%$ & $91 \%$ & NS & $88 \%$ & $93 \%$ & NS \\
\hline Gender (female) & $18 \%$ & $63 \%$ & $p<0.001$ & $55 \%$ & $47 \%$ & NS & $68 \%$ & $46 \%$ & $p=0.011$ \\
\hline Marital status (single) & $68 \%$ & $50 \%$ & $p=0.021$ & $43 \%$ & $59 \%$ & NS & $44 \%$ & $55 \%$ & NS \\
\hline
\end{tabular}


perceived by $83 \%$ and $90 \%$, respectively, as permitting private practice (Table 1). Furthermore, when students positively considering these two specialties as careers were compared to those not positively considering them, the former significantly more often rated the selection criterion, opportunity to practice privately, as important/ very important (Table 3). Conversely, family medicine was perceived by only $37 \%$ of respondents as permitting an opportunity for private practice (Table 3 ). Furthermore, only $34 \%$ of those considering the specialty rated an opportunity for private practice as an important/very important selection criterion versus $56 \%$ who were not considering it.

Another instance of such interaction was with the selection criterion "work only daytime hours" (Figure 2), reported as important/very important by $30 \%$ of the students. Ninety percent and $53 \%$ of students perceived that family medicine and pediatric specialists, respectively, had the possibility to "work limited hours". When students positively considering these two specialties as careers were compared to those who were not positively considering them, the former significantly more often rated the selection criteria, "work only daytime hours", as important/ very important (Table 3). Alternately, few students positively considering careers in general and orthopedic surgery perceived the specialties as being conducive to working limited hours ( $2 \%$ and $10 \%$ of students respectively), and were significantly less likely to consider the selection criteria "work only daytime hours" as important/very important than the rest of the students.

\section{Discussion}

This study provides Israeli healthcare leaders with information about how a group of Israeli medical students perceive six of the nine specialties with the greatest number of specialists [11]. The concrete opinions the students had about each of the six specialties elucidates medical specialty perception patterns prevalent among Israeli students, e.g. anesthesiology was universally unpopular among both genders and was overwhelmingly perceived as suffering from a workforce crisis. Moreover, this study provides further insights into these perceptions by simultaneously examining how the students' selection criteria matched their perceptions. When assessed from the marketing research perspective, these data provide the vendors (i.e. healthcare system administrators, department chairs and residency program directors) with objective information on whether certain aspects of a specialty's working conditions, remuneration, or clinical spectrum need to be changed to better align them with the students' selection criteria.

For instance, practically no students perceived general surgery as providing a controllable lifestyle or family time, which were reported as important/very important selection criteria by $69 \%$ and $80 \%$ of respondents, respectively. Additionally, $<7 \%$ opined that the specialty provides a reasonable relationship between lifestyle and income. Therefore, it is not surprising that $74 \%$ perceived general surgery as being plagued by a workforce crisis and reported that only $6 \%$ of their colleagues were considering it as a career.

These results reflect the notion that it is extremely difficult to attract potential buyers (students) to a product (specialty) they consider unappealing. In a market environment, unappealing products are usually modified to meet consumers' expectations, subjected to a different marketing/sales strategy, or withdrawn from the market [12]. The latter option is not relevant in the medical specialty arena. Therefore, when marketing the various specialties to medical students, the features perceived by them as causing some specialties to be unattractive might need modification to improve their appeal. Obviously, such changes may not be easily implemented but could be essential for specialties unable to attract sufficient students to their residency programs. For example, in the United States and other countries, efforts to modify the general surgery specialty have met with some successes in recruiting more residents, especially female ones $[13,14]$.

In many countries, primary care specialties, such as family medicine and pediatrics, are unpopular among medical students due to uncontrollable lifestyles and the poor relationship between lifestyle and income [15-17]. This situation is attributed to relatively low remuneration and solo practices, often in rural areas, leading practitioners to be at the beck and call of their patients [18]. In some of these countries anesthesiology is a popular specialty because it provides a controllable lifestyle with defined working hours and excellent remuneration [14,19]. The present study shows that in Israel the opposite is true. Specifically, family medicine was perceived as affording a controllable lifestyle, family time, and reasonable ratio of lifestyle to income along with the possibility of working limited hours. Pediatrics was similarly perceived. Both specialties were not considered to be experiencing workforce problems and were more attractive to female students than to male students, with $25 \%$ and $45 \%$ of female students positively considering careers in family medicine and pediatrics, respectively. This popularity is attributed to the characteristics of the Israeli medical system where such practitioners are largely employees of health maintenance organizations working fixed hours with minimal on-call duties and no in-patient responsibilities.

Anesthesiology was deemed by $97 \%$ of the respondents to be suffering a workforce crisis and by only $0.5 \%$ of respondents as being positively considered as a career by of their colleagues. Although anesthesiology was perceived as interesting and challenging by $43 \%$ of students, on par with pediatrics and orthopedic surgery, it was perceived 
by the majority of students as not providing a controllable lifestyle, high salary, the possibility of working limited hours, or allowing for family time. Furthermore, it was perceived as requiring long working hours and as having an unfavorable ratio of lifestyle to income. These results are not unexpected, given that Israeli anesthesiology is plagued by a perennial shortage of physicians leading to a vicious cycle of long working hours and many on-call duties, thereby further discouraging medical students from joining anesthesiology residency programs [20,21]. However, this study points to some features of the specialty that could be remediated in order to break this cycle. These include improved working conditions, compensation, and image. Corrective measures in other countries, aimed at similar issues, have successfully attracted medical students to the specialty [19].

There were distinct differences between how the student's perceived each of the six specialties (Table 1); however, within each of the specialties there were few disparities between the responses of male and female students (Table 2). As an example, 63\% of the respondents reported that pediatrics affords a controllable lifestyle, while only $8 \%$ reported that obstetrics/gynecology affords the same. Yet, in both instances there were no differences between the opinions of male and female students. In fact, most of the gender differences were in response to the questions of whether the specialty was boring or interesting and challenging. For example, a higher proportion of women perceived pediatrics and family medicine to be interesting and challenging, while a higher proportion of men thought that general and orthopedic surgery were interesting and challenging. Correspondingly, a higher proportion of women considered pediatrics and family medicine as specialties they were positively considering while a higher proportion of men were positively considering orthopedic and general surgery.

The importance of a specialty being interesting and challenging recurs throughout this study, with over $92 \%$ of the student's reporting it as being an important/very important selection criteria. Further, being interesting and challenging is the most consistently statistically significant perception when the opinions of those considering were compared to those not considering pursuing each of the 6 specialties (Table 3). However central "interest" is to selecting a career, it is a nebulous concept that is difficult to concretely define. Interest is a conglomeration of many factors including personality; intellectual ability and aptitude; manual skill; physical and emotional dispositions; and willingness to work in particular environments [3,22]. Interest also often correlates with gender, with men generally preferring to work with things and women with people $[23,24]$. Therefore, subsequent studies are needed to more deeply probe the determinants of interest as they relate to medical specialty selection and students' perceptions of the various specialties. Such studies promise to provide further insight into how specialty selections are made.

\section{Strengths and limitations}

This is among the few studies to examine Israeli medical students' perceptions of some of the healthcare system's key medical specialties. It provides both insight into the students' views of each of the specialties and how their selection criteria, perceptions, and interest in pursuing a specialty as a career interact. A limitation of this study is that since it measures perceptions, it is possible that students do not base answers on objective information but, rather, on impressions, biases, and incomplete information. However, it is very important to study perceptions, as they are the basis of many decisions in life. Moreover, we did not elicit the source of these perceptions to determine the basis on which they were formed, a topic that should be studied in the future. Finally, the questionnaires were anonymous so it was not possible to assess the characteristics of the non-responders. However, we do know that the male:female ratio of the responders reflects the male:female ratio of the medical school classes.

\section{Conclusions}

The Israeli healthcare system is continuously faced with the predicament of balancing the ever-increasing demand for a wide spectrum of clinical services with a sufficient supply of appropriately trained professionals to provide these services. However, the physician workforce is facing both an impending shortage of physicians and a maldistribution of physicians among the various specialties. The shortage has been officially recognized, leading to larger class sizes in existing medical schools and the opening of an additional medical school. However, only recently have some initiatives been taken to remedy the specialty distribution problem. Incentives were provided in the 2011 collective bargaining agreement which increased remuneration for specialties suffering workforce shortfalls, including general surgery and anesthesiology, and provided one-time monetary grants to those entering residencies in these specialties as well ongoing salary differentials. However, these initiatives were not based on objective data, but on the negotiators and their advisors opinions of what they thought would attract more medical students and interns to specialties with workforce shortages. This subjective approach is fraught with risks since it does not necessarily promise a solution to the problem. From the marketing research viewpoint, it is akin to developing a product without asking potential customers whether they would consider buying it.

The present study demonstrates the use of an objective approach to examining workforce problems, namely, how the selection criteria of the "consumers" match their perceptions of specific specialties. These results are the 
initial phase of a marketing research study and provide data for designing the next phase which will examine in greater detail the "customer's" positive and negative opinions of the "product". This second phase leads to further phases that elicit "customer's" opinions on proposed improved versions of the "product" that were designed using the previously obtained perceptions and opinions. This type of objective approach is generally unappealing to medical workforce administrators because it requires substantial personnel and expertise to perform the analyses. However, this type of research is important so that healthcare leaders can understand the younger generation's behavioral patterns and aspirations that focus on working conditions that contribute to controllable lifestyles and quality family time [25].

Besides demonstrating that gathering objective data on the opinions and perceptions of Israeli medical students provides a way to better understand physician workforce dynamics, this study also provides the Israeli healthcare leadership with a detailed assessment of Israeli medical students' perceptions of some of the major specialties. This information can be employed to tailor incentives to specifically attract students to specialties with workforce shortages. Workforce shortages in anesthesiology and general surgery are not surprising, given the poor opinions of those specialties reported from the students. These data provide evidence for the need for more than just one-time financial grants and small increases in salary to attract students to anesthesiology. It shows the need to improve the relationship of lifestyle to remuneration. This study also shows that the general surgery leadership needs to address the perceptions and realities that exist including, hard work, long hours, and an uncontrollable lifestyle. Finally, the information generated could also be used to design active interventions, such as marketing campaigns, to better recruit students to problematic specialties $[18,26]$. Studies similar to the current one should thus be performed on a regular basis using similar methodology to study other specialties in addition to the six included in the present study to assist in understanding residency selection trends among the medical students in all 5 of the Israeli medical schools.

\section{Endnotes}

${ }^{a}$ Internal Medicine was not chosen because it is usually a "pass through" residency i.e. a way-station on the way to sub-specialization. Only a minority of Israeli internal medicine residents do not sub-specialize. Therefore, the responses we would receive might not properly examine general internal medicine as a specialty but might also reflect the student's view of a possible subspecialty. We are in the process of developing a questionnaire to specifically study interest in general internal medicine vs interest in subspecialty training, but it is difficult to obtain a clear separation.
${ }^{b}$ The mathematical definition of reciprocal is inverse, opposite. In the present study reciprocal is used in factor analysis to designate a factor that relates in the opposite. For example, the manuscript relates that one factor was: "Working limited hours and family time. Long working hours was a reciprocal member". Therefore, working limited hours and long working hours were identified as being inversely related to each other. To clarify the issue we added the word "inverse" in parenthesis after reciprocal.

\section{Competing interests}

The authors declare that they have no competing interests.

\section{Authors' contributions}

CW concept, study design, data collection and analysis and manuscript writing. HT concept, questionnaire design, data collection, review and revision of manuscript. RYZR - study design, data collection and analysis, revision of manuscript. YGW questionnaire design, study design, data analysis and interpretation, review and revision of manuscript. UE study design, review and revision of manuscript. AA design, review and revision of manuscript. JES concept, study design, review and revision of manuscript. All authors read and approved the final manuscript.

\section{Acknowledgements}

The authors would like to thank the medical students who participated in this study. This research was funded by a grant from The Israel National Institute for Health Services and Health Policy Research.

\section{Author details}

${ }^{1}$ Department of Anesthesiology and Critical Care Medicine, HadassahHebrew University Medical Center, Hebrew University Hadassah School of Medicine, POB 12000, Jerusalem 91120, Israel. ${ }^{2}$ Department of Obstetrics and Gynecology, Hadassah-Hebrew University Medical Center, Hebrew University Hadassah School of Medicine, Jerusalem, Israel. ${ }^{3}$ Department of Orthopedic Surgery, Hadassah-Hebrew University Medical Center, Hebrew University Hadassah School of Medicine, Jerusalem, Israel. ${ }^{4}$ Henrietta Szold Hebrew University Hadassah School of Nursing, Jerusalem, Israel. ${ }^{5}$ Department of Family Medicine, School of Continuing Medical Education Ben Gurion University School of Medicine, Be'er Sheva, Israel.

Received: 24 January 2013 Accepted: 3 May 2013

Published: 21 May 2013

\section{References}

1. Sringer K, Kerpelman J, Skorikov V: Career preparation: a longitudinal, process-oriented examination. J Vocat Behav 2011, 79:158-169.

2. Borges NJ, Savicka MI: Personality and medical specialty choice: a literature review and integration. J Career Assessment 2002, 10:362-380.

3. Petrides KV, McManus IC: Mapping medical careers: questionnaire assessment of career preferences in medical school applicants and final-year students. BMC Med Educ 2004, 1:4-18.

4. Weissman C, Zisk-Rony RY, Schroeder JE, Weiss YG, Avidan A, Elchalal U, Tandeter $\mathrm{H}$ : Medical specialty considerations by medical students early during their clinical experience. Israel J Health Policy Res 2012, 1:13.

5. Weissman C, Schroder JE, Weiss Y, Elchalal U, Tandeter H, Zisk-Rony RY: Using marketing research concepts to investigate specialty selection by medical students. Med Educ 2012, 46:974-982.

6. Dorsey ER, Jarjoura D, Rutecki GW: The influence of controllable lifestyle and sex on the specialty choices of graduating U.S. medical students, 1996-2003. Acad Med 2005, 80:791-796.

7. Sanfey HA, Saalwachter-Schulman AR, Nyhof-Young JM, Eidelson B, Mann BD: Influences on medical student career choice: gender or generation? Arch Surg 2006, 141:1086-1094.

8. Dorsey ER, Jarjovra D, Rutecki GW: Influence of controllable lifestyle on recent trends in specialty choice by US medical students. JAMA 2003, 296:1173-1178.

9. Gierberg E: Gender similarities in doctors' preferences-and gender differences in final specialization. Soc Sci Med 2002, 54:591-605. 
10. Lambert TW, Goldacre MJ, Edwards C, Parkhouse J: Career preferences of doctors who qualified in the United Kingdom in 1993 compared with those of doctors qualifying in 1974, 1977, 1980, and 1983. BMJ 1996, 13:19-24.

11. Medical Manpower 2009. http://www.health.gov.l//download/docs/units/ meida/manpower2009/3.pdf. Accessed 30 December 2010.

12. Avlonitis GJ, Hart SJ, Tzokas NX: An analysis of product deletion scenarios. J Product Innovation Manag 2000, 17:41-56.

13. Miller $G$, Bamboat ZM, Allen F, et al: Impact of mandatory resident work hour limitations on medical students' interest in surgery. J Am Coll Surg 2004, 199:615-619.

14. Stablie BE: The surgeon: a changing profile. Arch Surg 2008, 143:345-350.

15. Jordan J, Brown JB, Russell G: Choosing family medicine: what influences medical students? Can Fam Phys 2003, 49:1131-1137.

16. Pagno PA, McGaha AL, Crosely PW, et al: Results of the 2010 national resident matching program: family medicine. Fam Med 2010, 42:552-561.

17. Morra DJ, Reghehr G, Ginsbirg S: Medical students, money, and career selection: students' perception of financial factors and remuneration in family medicine. Fam Med 2009, 41:105-110.

18. Lu DJ, Hakes J, Bai M, Tolhurst H, Dickinson JA: Rural intentions: factors affecting the career choices of family medicine graduates. Can Fam Phys 2008, 54:1016-1017.

19. Wass $C T$, Timothy $T R$, Randle DW, et al: Recruitment of house staff into Anesthesiology: a reevaluation of factors responsible for house staff selecting anesthesiology as a career and individual training program. J Clin Anesth 2003, 15:289-294.

20. Weissman C, Eidelman LA, Pizov R, Matot I, Klein N, Cohn R: The Israeli anesthesiology workforce. Isr Med Assoc 2006, 8:255-260.

21. Orbach-Zinger S, Rosenbum R, Svetsky S, Staiman A, Eidelman LA: Attitudes to anesthesiology residency among medical students in the American and the Israeli programs at the Sackler Faculty of Medicine, Tel Aviv University. Isr Med Assoc J 2011, 13:485-487.

22. Garcia-Sedeno M, Navarro Jl, Menacho I: Relationship between personality traits and vocational choice. Psychol Rep 2009, 105:633-642.

23. Su R, Rounds J, Armstrong PI: Men and things, women and people: a meta-analysis of sex differences in interests. Psychol Bull 2009, 135:859-884.

24. Correll SJ: Gender and the career choice process: the role of biased self-assessments. Am J Sociol 2001, 106:1691-1730.

25. Businger A, Viliger $P$, Sommer C, Furrer M: Argument for and against a career in surgery: a quantitative analysis. Ann Surg 2010, 252:390-396.

26. Leong FTL, Hardin EE, Gaylor M: Career specialty choice: a combined research-intervention project. J Voc Behavior 2005, 67:69-86.

doi:10.1186/2045-4015-2-19

Cite this article as: Weissman et al.: Israeli medical students' perceptions of six key medical specialties. Israel Journal of Health Policy Research 2013 2:19.

\section{Submit your next manuscript to BioMed Central and take full advantage of:}

- Convenient online submission

- Thorough peer review

- No space constraints or color figure charges

- Immediate publication on acceptance

- Inclusion in PubMed, CAS, Scopus and Google Scholar

- Research which is freely available for redistribution 